Check for updates

Cite this: RSC Adv., 2019, 9, 3493

Received 23rd November 2018 Accepted 21st January 2019

DOI: $10.1039 / c 8 r a 09651 a$

rsc.li/rsc-advances

\section{Influence of carbonization conditions on luminescence and gene delivery properties of nitrogen-doped carbon dots $\uparrow$}

\begin{abstract}
Claudel Mickaël,\$ Fan Jiahui, \$ Rapp Mickaël, Pons Françoise and Lebeau Luc (D) *
Carbon dots (CDs) have been intensively investigated due to their unique photoluminescence (PL) properties that are improved through surface passivation with nitrogen-containing groups. Recently, gene delivery applications emerged as passivation of CDs may yield positively charged nanoparticles that can interact with negatively charged nucleic acids. However previous work in the field focused on the use of high molecular weight polyamines for $C D$ passivation, posing the problem of the separation of nanoparticles from residual polymer that is harmful to cells. In this work, cationic CDs were prepared by pyrolysis of citric acid/bPEI600 (1/4, w/w) so unreacted low molecular weight reagents could be conveniently eliminated by extensive dialysis. Various reaction conditions and activation modes were evaluated and eleven CDs that exhibited superior solubility in water were produced. All the nanoparticles were characterized with respect to their physical, optical and PL properties and their ability to deliver plasmid DNA to mammal cells was evaluated. Despite their similar physical properties, the CDs displayed marked differences in their gene delivery efficiency. CDs produced under microwave irradiation in a domestic oven were revealed to be superior to all the other nanoparticles produced in this study and compared to the gold standard transfection reagent bPEI25k, with an optimal CD/pDNA w/w ratio that was significantly down shifted, as was the associated cytotoxicity.
\end{abstract}

\section{Introduction}

Carbon dots (CDs) are a carbon-dominated nanomaterial with a discrete and quasi-spherical structure, usually displaying oxygen- and nitrogen-containing functional groups. Since this newest member of the carbon nanoparticle family was accidentally discovered in $2004,{ }^{1}$ it has attracted considerable attention, due to its important properties, and has been the subject of more than five thousand reports. ${ }^{2}$ CDs are easily produced from inexpensive starting materials and using a significant number of various protocols, based on either topdown or bottom-up approaches. ${ }^{3-6}$ They are water soluble, chemically stable, biocompatible, and of low toxicity. They display photoluminescence (PL) properties, and are resistant to photobleaching. As a consequence, they became important in various fields such as optoelectronics, solar technology, photovoltaics, catalysis, biosensing, bioimaging, and drug delivery ${ }^{6-11}$ Most CDs are prepared with a passivated surface, with potential for further functionalization with organic and

Laboratoire de Conception et Application de Molécules Bioactives, UMR 7199 CNRS Université de Strasbourg, Faculté de Pharmacie, 74 route du Rhin-BP 60024, 67401 Illkirch, France. E-mail: llebeau@unistra.fr

† Electronic supplementary information (ESI) available. See DOI: 10.1039/c8ra09651a

\$ These authors contributed equally to this work. inorganic moieties, ${ }^{12}$ and due to their unique PL properties they are considered to be superior to quantum dots and organic dyes. ${ }^{13-15}$ In recent years, several groups started to focus their research on CD-based drug delivery and theranostic applications as CDs may afford the system multiple functions, including both delivery of therapeutics and fluorescence imaging. ${ }^{16-18}$ Especially, gene delivery applications became obvious as passivation of CDs with nitrogen-containing compounds most often produces positively charged nanoparticles. They may thus establish electrostatic interactions with negatively charged nucleic acids, resulting in the formation of nano sized complexes as required for cell internalization and further processing of the genetic material by the cellular machinery leading to (trans)gene expression or silencing. There are still limited reports on CD-based gene delivery systems and they may be classified according to the procedure used for the preparation of the carbon nanoparticles. Liu et al. who first described the use of CDs for intracellular gene delivery prepared the DNA nano-carrier by microwave-assisted pyrolysis of citric acid and branched polyethyleneimine $25 \mathrm{kDa}$ (bPEI25k). ${ }^{19}$ The obtained CDs allowed in vitro plasmid DNA (pDNA) transfection in the Cos-7 and Hep-G2 cell lines at a similar rate to that of parent bPEI25k, with lower associated cytotoxicity. Pierrat $e t$ al. developed similar CDs for airway administration and demonstrated their pDNA transfection efficiency both in vitro (A549 lung epithelial cell line) and in vivo (local administration in the 
mouse lung), ${ }^{\mathbf{2 0}}$ as well as their ability to deliver small interfering RNA (siRNA) in vitro. Recently, this CD-based delivery system was used for in vivo siRNA delivery and intratumoral injection of the complexes with a siRNA sequence targeting the vascular endothelial growth factor (VEGF) was shown to significantly inhibit tumor growth. ${ }^{21}$ Other groups produced analogous CDs using glycerol, ${ }^{22,23}$ poly(ethylene oxide), ${ }^{24}$ hyaluronic acid,${ }^{25}$ and glucose $^{26}$ in place of citric acid as the carbon source, and demonstrated transfection efficiency both in vitro and in vivo. Alternatively, the synthesis of CDs for gene delivery applications has also been carried out by various hydrothermal methods. Wu et al. reported the preparation of such CDs by heating bPEI25k at $200{ }^{\circ} \mathrm{C}$ for $10 \mathrm{~h} .{ }^{27}$ In this case, bPEI25k served both as the carbon source and the passivation reagent, and transfection efficiency was only qualitatively demonstrated in vitro. Other groups used various starting materials to produce the nanocarriers: glucose and bPEI25k, ${ }^{28}$ sodium alginate and hydrogen peroxide, ${ }^{29}$ folic acid and PEI1800, ${ }^{30}$ and hyaluronic acid and bPEI25k. ${ }^{31}$ Though the resulting CDs allowed pDNA intracellular delivery in vitro, the demonstration of in vivo efficacy is still awaited. All the nanoparticles described in these reports were hydrophilic, and were used as produced to transfect cells. A very recent report by Yu et al. described the synthesis of amphiphilic nanoparticles obtained through the grafting of dodecyl chains onto hydrophilic CDs prepared from PEI600 by a solvothermal method. ${ }^{32}$ The amphiphilic nano carriers were able to deliver pDNA and siRNA to A549 and A549-Luc cells. Finally, besides these bottom-up approaches to prepare CDs with transfection capabilities, one top-down procedure has been reported that involved laser ablation of a carbon target, and post-treatment with alkyl-PEI2k at $120{ }^{\circ} \mathrm{C} .{ }^{33}$ In siRNA delivery experiments, 30-40\% gene silencing could be achieved in vitro. Intratumoral administration of alkyl-PEI $2 \mathrm{k}-\mathrm{CD} / \mathrm{pDNA}$ complexes resulted in high transgene expression in the tumor tissue, evidencing the in vivo potency of this carrier.

As mentioned above, all the CD-based gene delivery systems described to date involved the carbonization of a high molecular weight polymer (PEI, PEG, hyaluronic acid, alginate...), either as the passivation agent or as the carbon source, or both. Purification of the nanoparticles produced during this process invariably involves a dialysis step. Proper elimination of the residual polymer probably is not possible, even using dialysis membranes with a high molecular weight cut-off (MWCO), which essentially results in massive loss of the material of interest. Furthermore, residual starting aminated polymeric materials most of the time display intrinsic transfection properties, making uncertain accurate interpretation of the experimental data. With this in mind, we introduced consequential modifications in the popular one-pot carbonization processing of citric acid-PEI precursor mixtures to produce CDs. Especially, we investigated the possibility to produce cationic CDs from citric acid and low molecular weight branched PEI, bPEI600, such as unreacted polymer can be efficiently removed by dialysis on membranes with medium MWCO. Besides, contrarily to higher molecular weight PEI, bPEI600 cannot promote pDNA or siRNA intracellular delivery, presumably in relation with the poor stability of the complexes it forms with nucleic acid. Finally, bPEI600 is non-toxic to cells, which is not the case for higher molecular weight PEI. ${ }^{34}$ Various carbonization procedures were evaluated for the production of the nanoparticles, including solvothermal treatment, conventional pyrolysis under atmospheric pressure, and microwave-assisted pyrolysis. The CDs produced were characterized and compared with each other for their capacity to promote pDNA transfection in mammalian cells. We found out that microwaveassisted pyrolysis under non-solvothermal conditions provided CDs with superior transfection properties when compared to the other activation protocols used in this study.

\section{Experimental}

\subsection{Reagents and chemicals}

Citric acid, bPEI25k, bPEI600, and 3-(4,5-dimethylthiazol-2-yl)2,5-diphenyl tetrazolium bromide (MTT) were from SigmaAldrich (St Louis, MO, USA). Dialysis membranes were from Spectrum Laboratories (Rancho Dominguez, CA, USA). A549 cells (human lung carcinoma; CCL-185) were obtained from ATCC-LGC (Molsheim, France). Plasmid pCMV-GLuc (5.7 kbp, Nanolight Technology, Pinetop, Az, USA) was used as reporter gene to monitor in vitro DNA transfection activity. This plasmid encodes the Gaussia princeps luciferase gene. ${ }^{35}$ pDNA concentration refers to phosphate content. Fetal bovine serum (FBS), culture media (Dulbecco's Modified Eagle Medium, DMEM) and supplements were from GIBCO-BRL (Cergy-Pontoise, France). Coelenterazine was from Alfa Aesar (Biesheim, France). All CDs and buffer solutions were prepared with deionized water purified with an EMD Millipore Milli-Q $\mathrm{Q}^{\mathrm{TM}}$ integral system (resistivity $\leq 18.2 \mathrm{M} \Omega \mathrm{cm}$ ) and filtered through a $0.2 \mu \mathrm{m}$ polycarbonate membrane.

\subsection{Synthesis of CDs}

Solvothermal preparations. Citric acid (1.00 g), bPEI600 (4.00 $\mathrm{g})$, and $\mathrm{H}_{2} \mathrm{O}(50 \mathrm{~mL})$ were mixed under vigorous stirring to form a homogeneous solution. The solution was then transferred into a reaction vessel fitted with a reflux condenser, and heated for $24 \mathrm{~h}$ at $100{ }^{\circ} \mathrm{C}$ (protocol A1). For higher temperature reaction, the reaction mixture (citric acid: $0.125 \mathrm{~g}$; bPEI600: $0.500 \mathrm{mg}$; $\mathrm{HCl} 0.1 \mathrm{~N}: 5 \mathrm{~mL}$ ) was transferred into a tightly sealed $10 \mathrm{~mL}$ borosilicate glass vial and the temperature was raised to and maintained at $180^{\circ} \mathrm{C}$ for $4 \mathrm{~h}$ by conventional heating using an Anton Paar Monowave 50 synthesis reactor (protocol A2). When cooled down to room temperature, the resulting colored solutions were transferred into a dialysis bag (Spectra/Por 3, MWCO $1000 \mathrm{Da}$ ) and were equilibrated for $12 \mathrm{~h}$ against $1 \mathrm{~L} \mathrm{HCl}$ $0.1 \mathrm{~N}$ (dialysate was replaced at 1, 3, $7 \mathrm{~h}$ ), and then against ultrapure water for an additional 12 h-period. Finally the brownyellow solutions were filtered through a $0.22 \mu \mathrm{m}$ PES membrane (Millex) and freeze-dried at $-50{ }^{\circ} \mathrm{C}$ for $24-36 \mathrm{~h}$. Protocols A1 and A2 yielded $0.662 \mathrm{~g}$ (CD1) and $0.020 \mathrm{~g}$ (CD2) of hygroscopic powdered yellow and brown-yellow materials, respectively.

Pyrolysis under conventional heating and solvent-free conditions. Citric acid (0.125 g) and bPEI600 (0.500 g) were 
introduced into a $10 \mathrm{~mL}$ borosilicate glass vial without solvent and heated for $30 \mathrm{~min}$ in a Monowave 50 synthesis reactor (protocol B1: $180{ }^{\circ} \mathrm{C}$; protocol B2: $210{ }^{\circ} \mathrm{C}$; protocol B3: $225{ }^{\circ} \mathrm{C}$ ). The colored residue was vigorously stirred with $\mathrm{HCl} 1 \mathrm{~N}$, centrifuged (10.000 rpm, $5 \mathrm{~min}$ ), and the supernatant was dialyzed and freeze-dried as described above. Protocols B1, B2, and B3 yielded $0.410 \mathrm{~g}$ (CD3), $0.267 \mathrm{~g}$ (CD4), and $0.415 \mathrm{~g}$ (CD5) of brown hygroscopic powdered materials, respectively.

In another protocol (protocol B4), citric acid (1.25 g) and bPEI600 (5.00 g) were mixed in a $25 \mathrm{~mL}$ borosilicate reaction flask without solvent and heated at $180{ }^{\circ} \mathrm{C}$ for 30 min under atmospheric pressure. During this first heating step, volatile was integrally driven out of the reaction vessel. Then temperature was raised to and maintained at $230{ }^{\circ} \mathrm{C}$ for an additional period of $30 \mathrm{~min}$. The resulting reaction mixture was treated as described above to yield $0.786 \mathrm{~g}$ of a black hygroscopic powdered material (CD6).

Preparations under microwave irradiation. Citric acid $(1.00$ $\mathrm{g}$ ), bPEI600 (4.00 g), and $\mathrm{H}_{2} \mathrm{O}(10 \mathrm{~mL})$ were mixed under vigorous stirring to form a homogeneous solution. The solution was then transferred into a tightly sealed borosilicate glass vial and heated for $30 \mathrm{~min}$ at $100{ }^{\circ} \mathrm{C}$ using an Anton Paar Monowave 500 microwave reactor (protocol $\mathrm{C}$ ). The crude residue was purified by dialysis and yielded $0.216 \mathrm{~g}$ of yellow powdered material upon freeze-drying (CD7).

Citric acid (0.125 g) and bPEI600 (0.500 g) were introduced in a tightly sealed borosilicate glass vial without solvent and heated at the indicated temperature for $180 \mathrm{~s}$ using an Anton Paar Monowave 500 microwave reactor (protocol D1: $180{ }^{\circ} \mathrm{C}$; protocol D2: $210{ }^{\circ} \mathrm{C}$; protocol D3: $225{ }^{\circ} \mathrm{C}$ ). The crude residues were purified by dialysis and yielded $0.263 \mathrm{~g}$ (CD8), $0.313 \mathrm{~g}$ (CD9), and $0.249 \mathrm{~g}$ (CD10) of brown powdered materials upon freeze-drying, respectively.

Citric acid (0.125 g), bPEI600 (0.500 g), and $\mathrm{HCl} 0.1 \mathrm{~N}(5 \mathrm{~mL})$ were mixed in an Erlenmeyer flask to form a homogeneous solution that was heated in a domestic microwave oven at $620 \mathrm{~W}$ for $180 \mathrm{~s}$ (protocol E). Noteworthy, using temperature measuring strips, the maximum temperature reached in the vessel was determined to be between 249 and $254{ }^{\circ} \mathrm{C}$. The residue was stirred with $\mathrm{HCl} 1 \mathrm{~N}(5 \mathrm{~mL})$ for $15 \mathrm{~min}$, centrifuged, and the supernatant was dialyzed as described above to yield $0.194 \mathrm{~g}$ of a brown hygroscopic powdered material (CD11).

\subsection{Characterization of CDs}

NMR spectroscopy. NMR spectra from samples prepared in deuterium oxide were recorded on a Bruker $400 \mathrm{MHz}$ Avance III instrument. Chemical shifts $\delta$ are reported in ppm relative to water residual peak as an internal reference (HDO at $4.78 \mathrm{ppm}$ ).

Infrared spectroscopy. Fourier transform infrared spectroscopy (FT-IR) was performed on dry samples, on a FT-IR Nicolet 380 spectrometer in the attenuated total reflectance (ATR) mode.

Elemental analysis. The elemental composition of the CDs was determined by analysis on an Elementar Vario EL III apparatus.
UV-visible and fluorescence measurements. CD samples were prepared in ultra-pure water $\left(0.10 \mathrm{mg} \mathrm{mL}{ }^{-1}\right)$. Absorption spectra were recorded on a UviKon XL spectrometer (Bio-Tek Instruments) using a $1 \mathrm{~mL}$ quartz cuvette. Fluorescence spectra were recorded on a Fluoromax-4 spectrofluorometer (Horiba Scientific).

Quantum yield measurements. Fluorescence quantum yields were determined using coumarin $343\left(\mathrm{c} 343, \Phi_{\mathrm{s}}=0.63\right.$ (ref. 36)) as a standard. The fluorescence quantum yield of the CDs was calculated according to

$$
\Phi_{\mathrm{CD}}=\Phi_{\mathrm{s}}\left(F_{\mathrm{CD}} / F_{\mathrm{s}}\right)\left(A_{\mathrm{s}} / A_{\mathrm{CD}}\right)
$$

where $\Phi_{\mathrm{CD}}$ and $\Phi_{\mathrm{s}}$ are the quantum yields of the CDs and the standard (resp.), $F_{\mathrm{CD}}$ and $F_{\mathrm{S}}$ are the measured integrated emission intensities, and $A_{\mathrm{CD}}$ and $A_{\mathrm{s}}$ are the absorbances. In order to minimize fluorescence quenching, absorbance in the $10 \mathrm{~mm}$ fluorescence cuvette was kept below 0.10 at the excitation wavelength.

\subsection{Dynamic light scattering measurements}

The average particle size and zeta potential ( $\zeta)$ of the CDs were measured by dynamic light scattering (DLS) using a Zetasizer NanoZS apparatus (Malvern Instruments, Paris, France). All measurements were performed on $1.0 \mathrm{mg} \mathrm{mL}{ }^{-1}$ samples in $1.5 \mathrm{mM} \mathrm{NaCl}, \mathrm{pH} \mathrm{7.4}$, at $25{ }^{\circ} \mathrm{C}$ and in triplicate. Data were analyzed using the multimodal number distribution software supplied with the instrument and expressed as mean $( \pm \mathrm{SD})$.

\subsection{Preparation of $\mathrm{CD} / \mathrm{pDNA}$ complexes}

The pDNA complexes formulated at various weight ratios were prepared by mixing equal volumes of CDs and pDNA solutions (prepared at the adequate concentration in ultra-pure water). The complexes were allowed to form for $30 \mathrm{~min}$ at room temperature without handling. Finally, the mixture was homogenized by pipetting up and down and subsequently used for in vitro transfection experiments. PEI/pDNA complexes were prepared similarly, from bPEI25k or bPEI600 solutions at adequate concentration.

\subsection{Cell culture}

A549 cells were grown in culture flasks at $37{ }^{\circ} \mathrm{C}$ in a $5 \% \mathrm{CO}_{2}$ humidified chamber using DMEM-F12 medium containing FBS (10\%), L-glutamine (2 mM), penicillin (100 units per $\mathrm{mL}$ ), streptomycin $\left(100 \mu \mathrm{g} \mathrm{mL}^{-1}\right)$, and Hepes ( $\left.5 \mathrm{mM}\right)$. At confluency, cells were released with trypsin, centrifuged $\left(4{ }^{\circ} \mathrm{C}, 5 \mathrm{~min}, 120 \mathrm{~g}\right)$, resuspended in culture medium, and counted before seeding in culture flasks (sub-culturing) or plates (transfection experiment).

\subsection{Transfection assay}

A549 cells were seeded into 96-well culture plates (BectonDickinson) at a density of 6000 cells per well in $100 \mu \mathrm{L}$ complete culture medium. Twenty-four hours later, freshly prepared CD/pCMV-GLuc complexes (typically $10 \mu \mathrm{L}$ containing 
$0.4 \mu \mathrm{g}$ pDNA) were added to each well of the plates. Then, cells were let to grow in the incubator without further handling for 24 h. Then, Gaussia luciferase production was measured by monitoring light production on an aliquot of culture supernatant $\left(20 \mu \mathrm{L}\right.$ of a $1 / 100^{\text {th }}$ dilution of supernatant prepared in nonsupplemented culture medium) for $1 \mathrm{~s}$ upon addition of the coelenterazine substrate $(50 \mu \mathrm{L}, 1.5 \mu \mathrm{M})$ using a luminometer (Berthold Centro LB960 XS, Thoiry, France). Value for each sample is the mean of a triplicate determination $( \pm \mathrm{SD})$.

\subsection{Cytotoxicity assay}

Mitochondrial activity measurements (MTT assay) were used to assess the cytotoxicity of $\mathrm{CD} / \mathrm{pDNA}$ complexes in transfection experiments. After incubation of complexes and culture medium removal for Gaussia luciferase assay, cells were carefully washed with PBS before addition of complete culture medium containing $0.5 \mathrm{mg} \mathrm{mL} \mathrm{m}^{-1}$ MTT $(100 \mu \mathrm{L}$ per well). After MTT incubation for $1 \mathrm{~h}$ at $37^{\circ} \mathrm{C}$, culture medium was removed and cells were lysed with DMSO $(100 \mu \mathrm{L})$. Intensity of MTT reduction was evaluated by measuring absorbance of the resulting solution at $570 \mathrm{~nm}$. Viability of treated cells was expressed as the percentage of the absorbance measured in untreated cells. Value for each sample is the mean of triplicate determinations $( \pm \mathrm{SD})$.

\subsection{Transmission electron microscopy}

Micrographs were obtained using a benchtop transmission electron microscope (TEM) operating at $5 \mathrm{kV}$ (LVEM5, Cordouan Technologies, Pessac, France). Carbon-coated grids ( $\mathrm{Cu}$ 300HD, Pacific Grid Tech, San Francisco, USA) were glow discharged at $90 \mathrm{~V}$ and $2 \mathrm{~mA}$ for $15 \mathrm{~s}$ before deposition of the CD sample $\left(0.5 \mu \mathrm{L}, 1.0 \mathrm{mg} \mathrm{mL}^{-1}\right)$. The grids were allowed to dry at room temperature for at least $2 \mathrm{~h}$ before observation. Average size of the particles was determined by image analysis using the Image J software (v 1.50i, NIH, USA), from a set of 300-1000 particles.

\section{Results and discussion}

\subsection{Synthesis of CDs}

The one-pot carbonization processing of citric acid and PEI as precursors has been especially popular in many publications describing the preparation of CDs. ${ }^{19-21,37-42}$ This is likely due to the easy implementation of the synthetic protocol, such as simple heating of precursors at high temperature $\left(180-250{ }^{\circ} \mathrm{C}\right)$ for a few hours. Though the synthesis has almost become a "classic" in the preparation of CDs, reports are disparate in regards to the yield of the preparations and to the properties (size, zeta potential, fluorescence spectral features) of the nanoparticles that are produced. Consequently, there are questions on the structural differences between the CDs produced under various experimental conditions. We thus carried out various protocols to produce CDs from a mixture of citric acid-bPEI600 (1/4, w/w) and investigated their properties. Solvothermal preparations involved heating in a reaction vessel fitted with a reflux condenser $\left(T=100{ }^{\circ} \mathrm{C}\right.$, ambient pressure $)$ for
$24 \mathrm{~h}$ (protocol A1) or in a tightly sealed synthesis reactor $(T=$ $180{ }^{\circ} \mathrm{C}, 4 \mathrm{~h}$, protocol A2). Pyrolysis under conventional heating of a solvent-free mixture of citric acid and bPEI600 under ambient pressure for $30 \mathrm{~min}$ was achieved at 180, 210, and $225{ }^{\circ} \mathrm{C}$ (protocols B1, B2, and B3, resp.). Alternatively, the mixture of citric acid and bPEI600 was heated at $180{ }^{\circ} \mathrm{C}$ under ambient pressure for $30 \mathrm{~min}$, and at $230{ }^{\circ} \mathrm{C}$ for an additional $30 \mathrm{~min}$ period (protocol B4).

Microwaves provide a dielectric heating method with many unique characteristics (e.g., shorter reaction time, easy process control, low energy consumption, and absence of a temperature gradient) that are important in reactions with multiple precursors, because the different thermolytic reactions must be controlled simultaneously through rapid heating rates and uniform heating. Pyrolyses under microwave irradiation were thus carried out in a Monowave 500 reactor (Anton Paar) in water at $100{ }^{\circ} \mathrm{C}$ for $30 \mathrm{~min}$ (protocol $\mathrm{C}$ ), or in solvent-free conditions at 180,210 , and $225^{\circ} \mathrm{C}$ for $3 \mathrm{~min}$ (protocols D1, D2, and D3, resp.). Additionally, citric acid and bPEI600 in water were heated in a domestic microwave oven operated at $620 \mathrm{~W}$ for $3 \mathrm{~min}$ (protocol E). Additional details are provided in the Experimental section. All the nanoparticles produced were purified by extensive dialysis using a membrane with a MWCO of $1000 \mathrm{Da}$. The amounts of nanoparticles (ratio of the amount of isolated CDs to that of starting materials, in \%) obtained after freeze-drying of the dialysis bag content are reported in Table 1. As can be observed, the yield of CDs prepared according to the various protocols is highly variable, spreading from only a few percent (protocols A2 and C) to 50-65\% (protocols B1, B2, B3, and D2). Although data interpretation is not straightforward, some trends yet are discernable. Solvothermal reactions invariably furnished CDs in a low yield, i.e., 4 to $11 \%$ (protocols $\mathrm{A} 1, \mathrm{~A} 2$, and $\mathrm{C})$ whatever the reaction temperature $\left(100\right.$ or $\left.180^{\circ} \mathrm{C}\right)$ or heating mode (conventional or microwave heating). At the opposite, solvent-free pyrolysis led to high yields when considering this type of synthetic work (protocols B1-B3 and D1-D3). On the other hand, overheating of the reagents in solvent-free conditions was counter-productive (protocol B4), probably

Table 1 Characteristics of the CDs produced according to the various protocols

\begin{tabular}{llrlrl}
\hline & & \multicolumn{3}{l}{ Size $^{b}(\mathrm{~nm})$} \\
\cline { 4 - 5 } & & & \multicolumn{2}{l}{} \\
Sample & Protocol & Yield $^{a}(\%)$ & \multicolumn{1}{l}{ DLS } & TEM & $\zeta(\mathrm{mV})$ \\
\hline CD1 & A1 & 11 & $241.4 \pm 38.6$ & 14.1 & $+22.5 \pm 4.7$ \\
CD2 & A2 & 3 & $20.7 \pm 2.1$ & 15.6 & $+32.0 \pm 3.7$ \\
CD3 & B1 & 66 & $11.4 \pm 1.5$ & 14.1 & $+37.5 \pm 2.1$ \\
CD4 & B2 & 43 & $10.8 \pm 0.6$ & 13.2 & $+40.7 \pm 1.8$ \\
CD5 & B3 & 65 & $12.2 \pm 0.8$ & 16.9 & $+37.6 \pm 2.6$ \\
CD6 & B4 & 13 & $15.3 \pm 1.0$ & 14.2 & $+26.8 \pm 2.6$ \\
CD7 & C & 4 & $29.4 \pm 3.3$ & 16.5 & $+26.7 \pm 1.0$ \\
CD8 & D1 & 42 & $13.8 \pm 0.4$ & 16.2 & $+28.7 \pm 1.5$ \\
CD9 & D2 & 50 & $20.3 \pm 0.7$ & 18.9 & $+27.3 \pm 2.4$ \\
CD10 & D3 & 40 & $11.0 \pm 0.2$ & 15.2 & $+27.3 \pm 1.5$ \\
CD11 & E & 31 & $20.6 \pm 3.6$ & 9.3 & $+33.3 \pm 1.8$
\end{tabular}

${ }^{a}$ Calculated as the ratio of the amount of isolated CDs to that of starting material (citric acid and bPEI600). ${ }^{b}$ As determined by DLS or TEM. 
because of the formation of large aggregates or charcoal that were not water-soluble anymore. Last, reactions under microwave irradiation at ambient pressure (i.e. with exclusion of volatiles) provided CDs with an intermediate yield (31\%, protocol E). This is consistent with the solvent-free conditions met at the end of the 3 min heating period.

\subsection{Characterization of CDs}

The size and zeta potential ( $\zeta$ ) of the CDs here above produced have been determined by DLS and TEM (Table 1). As the CDs were supposedly decorated at their periphery with titratable amine groups (originating from the PEI chains), all measurements were performed at a fixed and biologically relevant $\mathrm{pH}$ value, i.e. $\mathrm{pH}$ 7.4. For DLS analyses, the samples were prepared in $1.5 \mathrm{mM} \mathrm{NaCl}$ ( $\mathrm{pH} 7.4$ ) for proper determination of $\zeta$ potential. Analysis of multiple TEM images yielded an average dot size for each sample (Fig. 1). DLS and TEM provided consistent results, in most cases, with particle sizes in the $10-20 \mathrm{~nm}$ range (Table 1). One exception was observed for CD1 prepared according to protocol A1. In this case, DLS overestimated a population of aggregates (presumably "polymer" $\operatorname{dots}^{\mathbf{4 3 , 4 4}}$ ), as intensity of scattering is proportional to the diameter of the particles raised to the sixth power (from Rayleigh's approximation). As expected, the CDs revealed highly positively charged with $\zeta$ values ranging from +22.5 to $+40.7 \mathrm{mV}$. Pyrolysis under solvent-free conditions and conventional heating provided the more cationic nanoparticles (CD3-CD5), while overheating resulted in a gradual decrease in the $\zeta$ value (CD6).

The ${ }^{1} \mathrm{H}$-NMR spectra for the various CDs were recorded in $\mathrm{D}_{2} \mathrm{O}$ (Fig. 2 and ESI, Fig. $\mathrm{S} 1 \dagger$ ). The signals for all protons appeared significantly broadened in comparison with those in free bPEI600 and citric acid molecules as is exemplified for CD4 in Fig. 2. This broadening effect is consistent with the attachment to large species of lower mobility, thus inducing slower isotropic averaging ${ }^{45}$ and also with the inhomogeneous distribution of sites at the surface of the carbon nanoparticles. ${ }^{46}$ For CDs produced via a low temperature process (CD1 and CD7, prepared at $100{ }^{\circ} \mathrm{C}$ ), the presence of a characteristic $\mathrm{AB}$ system at 2.8-2.9 ppm (ESI, Fig. S1, $\uparrow$ indicated by arrows) still suggested the existence of citrate moieties or related methylene groups $\left(\mathrm{CH}_{2} \mathrm{COOH}\right)$ at the surface of the nanoparticles. For all

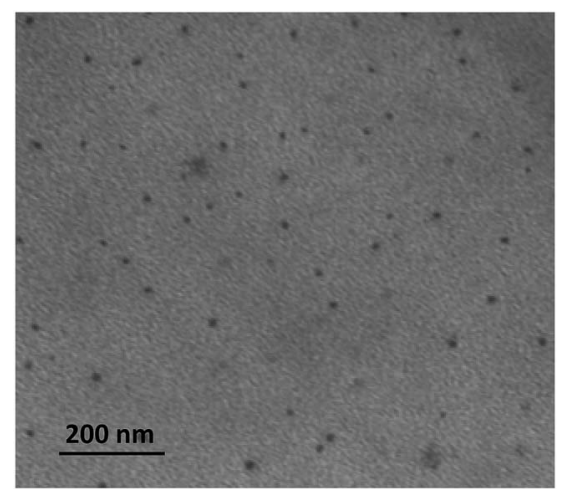

Fig. 1 Representative TEM image of the CDs synthesized from citric acid and bPEI600 (CD4).

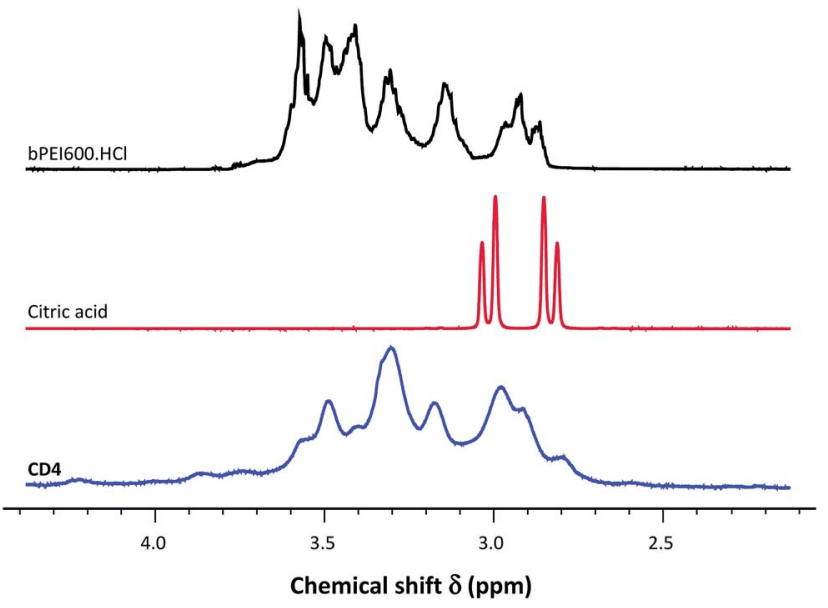

Fig. $2{ }^{1} \mathrm{H}$-NMR spectra of bPEI600-passivated CDs (CD4), and control spectra obtained for citric acid and bPEI600. Spectra for the other CDs are provided as ESI. $\dagger$

other CDs, except CD6, NMR spectra displayed the same features and exactly superimposed which did suggest that these CDs showed no measurable difference with regards to their surface decoration. The two-step pyrolysis processing involved in the preparation of CD6 and the higher temperature the nanoparticles faced led to a significantly modified NMR pattern where the previously observed signals were mainly lost (aromatization) or shifted downfield.

To gain structural insight about the surface state of the CDs (presence of functional groups on the surface) FT-IR spectroscopy was further employed (Fig. 3 and ESI, Fig. S2 $\dagger$ ). The broad peak centered at $3380 \mathrm{~cm}^{-1}$ was attributed to the $\mathrm{O}-\mathrm{H}$ and $\mathrm{N}-\mathrm{H}$ vibrational stretch of the carboxylic moieties (acid and amide) and residual water. The absorptions around 2950 and $2850 \mathrm{~cm}^{-1}$ represented $\mathrm{C}-\mathrm{H}$ symmetric and antisymmetric stretching that came from the PEI moieties immobilized at the surface of the nanoparticles. The carboxylic acid groups in citric acid that are featured by strong absorption at $1740-1680 \mathrm{~cm}^{-1}$ remained prominent in the spectra of the CDs, suggesting significant presence of carbonyl moieties in the nanoparticles.

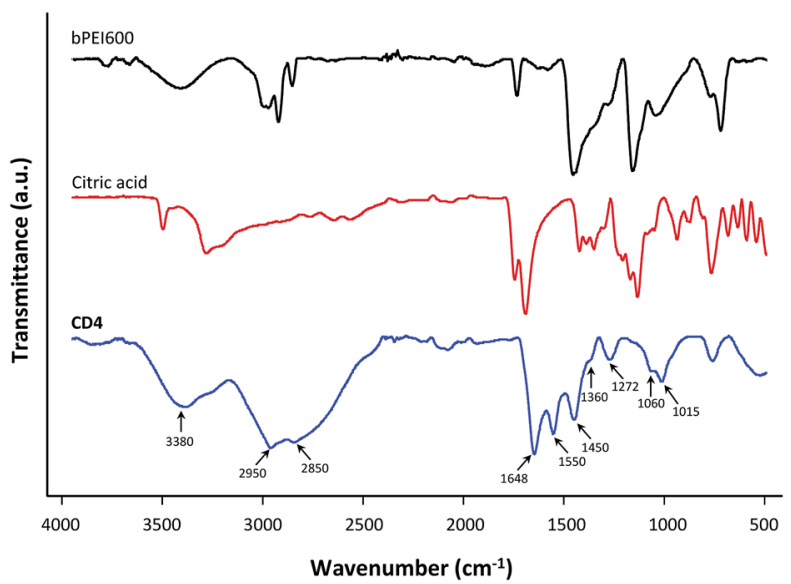

Fig. 3 FT-IR spectra of CD4, and control spectra obtained for citric acid and bPEI600. Spectra for the other CDs are provided as ESI. $\uparrow$ 
The absorption band at $1648 \mathrm{~cm}^{-1}$ thus corresponded to the stretching vibration of $\mathrm{C}=\mathrm{O}$ band (amide $\mathrm{I}$ band), which could be ascribed to the typical vibration of grafted $\mathrm{O}=\mathrm{CNH}$ functionalities at the surface of the nanoparticles. ${ }^{47}$ The peaks at 1550 and $1360 \mathrm{~cm}^{-1}$ were attributed to the asymmetric and symmetric carboxylate stretch. ${ }^{48}$ The one at $1450 \mathrm{~cm}^{-1}$ corresponded to stretching of $\mathrm{C}-\mathrm{N}=$ in the aromatic core of the nanoparticles. A peak at $1272 \mathrm{~cm}^{-1}$ was tentatively assigned to the $\mathrm{C}-\mathrm{N}$ stretching of PEI. The peaks at 1060 and $1015 \mathrm{~cm}^{-1}$ were attributed to stretching vibrations of $-\mathrm{OH}$ and $-\mathrm{NH}$. The characteristic $\mathrm{N}-\mathrm{H}$ and $\mathrm{C}-\mathrm{H}$ absorptions at 3500-3100 and 2950-2800 $\mathrm{cm}^{-1}$, respectively, observed for bPEI600 were not much attenuated in the dot samples thus revealing that precursor PEI was significantly spared under the various carbonization procedures used in this work. This is in agreement with the summary picture of the one-pot processing that citric acid, as the main carbon source, is more for being carbonized into de nanoparticle core, and PEI more for the surface passivation of the resulting CDs. No significant differences were observed when comparing FT-IR spectra for most of the prepared CDs (CD3-CD5, CD8-CD11). In the CDs prepared at the lower temperature (CD1 and CD7), the peak at $1718 \mathrm{~cm}^{-1}$ corresponding to the $\mathrm{C}=\mathrm{O}$ stretching vibration in citric acid was still evident revealing partial carbonization of the carbon source. Correlatively, only a nascent absorption could be observed at $1648 \mathrm{~cm}^{-1}$ (amide $\mathrm{C}=\mathrm{O}$ stretching). This was as well observed for CD2 that was prepared by short solvothermal treatment of the starting materials at an intermediate temperature $\left(4 \mathrm{~h}\right.$ at $\left.180^{\circ} \mathrm{C}\right)$. In this case however, the peak at $1718 \mathrm{~cm}^{-1}$ was no more visible, revealing that extended carbonization of citric acid occurred with formation of amides only in reduced amount. Finally, FT-IR spectrum for CD6 featured stronger absorption bands at $1360 \mathrm{~cm}^{-1}$ (C-N stretching of PEI) and $1270 \mathrm{~cm}^{-1}$ (related to different modes of $\mathrm{C}-\mathrm{O}-\mathrm{C}$ or $\mathrm{C}-\mathrm{O}$ stretching). This suggested that moderate overheating of the CD sample had significant impact on the nature of the chemical groups in the nanoparticles. With the aim to get some additional information on the structure of the CDs, elemental analyses were performed and the carbon, nitrogen, and hydrogen contents of the nanoparticles were determined (Table $\mathrm{S} 1 \dagger)$. No distinct trend emerged and the variations in the CDs composition could hardly be related to the pyrolysis conditions (reaction temperature or duration), except that the CDs with the higher carbon content (CD6, CD10, and CD11) were those produced under the harsher reaction conditions $\left(T>225{ }^{\circ} \mathrm{C}\right)$. This is consistent with the formation of extended polycyclic aromatic hydrocarbon (PAH) domains at high temperature. Interestingly, nitrogen content of the CDs did not correlate with the $\zeta$ value as would have been primarily expected. This indicated that nitrogen distribution at the surface and within the core of the particles may significantly vary from one CDs sample to another, "internal" nitrogen atoms (i.e., those in the nanoparticle core) and pyridinic ones less contributing to the net positive charge of the nanoparticles than graphitic ones displayed at the surface.

The optical properties of the CDs were investigated (Table 2, Fig. S3 $†$ ). All the nanoparticles displayed UV-vis absorption spectrum with two peaks around 250 and $360 \mathrm{~nm}$ and extending to $600 \mathrm{~nm}$ without noticeable structures. The two main peaks were ascribed to $\pi \rightarrow \pi^{*}$ transition of aromatic $\mathrm{C}=\mathrm{C}$ bonds and to $\mathrm{n} \rightarrow \pi^{*}$ electronic transition of $\mathrm{C}-\mathrm{N} / \mathrm{C}=\mathrm{N}$ and $\mathrm{C}-\mathrm{O} / \mathrm{C}=\mathrm{O}$ structures, respectively. ${ }^{49}$ The second peak was accompanied with a shoulder at $315 \mathrm{~nm}$ for most of the CDs, except those prepared at low temperature. For easier comparison between CDs, absorptivity in the material samples was characterized by a mass attenuation coefficient, $\varepsilon_{\mathrm{m}}$, expressed in $\mathrm{L} \mathrm{g}^{-1} \mathrm{~cm}^{-1}$, determined for the maximum absorption around $360 \mathrm{~nm}$. As can be observed, increasing the temperature and/or duration of the reaction yielded particles with higher $\varepsilon_{\mathrm{m}}$ values, which is consistent with the image of a temperature- and time-controlled carbonization during the pyrolysis reactions leading to the formation of $\mathrm{sp}^{2}$-hybridized aromatic domains. Thus, CD7 that combined low temperature $\left(100{ }^{\circ} \mathrm{C}\right)$ and short reaction time $(30 \mathrm{~min})$ offered the lower $\varepsilon_{\mathrm{m}}$ value in the sample series, and intermediate results were obtained for CD1 that experienced a longer heating period (24 h) at the same temperature. In the CD3-CD6 sample series, $\varepsilon_{\mathrm{m}}$ increased gradually from 1.71 to 5.04 while the temperature increased from 180 to $230^{\circ} \mathrm{C}$. The same was observed for the CD8-CD10 sample series, with measured $\varepsilon_{\mathrm{m}}$ values ranging from 1.83 to 2.44 . Though the reaction times were different, the same trend was still experienced in CDs prepared by solvothermal reaction under conventional heating (see CD1 and CD2).

Table 2 Photoluminescence properties of the as-prepared CDs

\begin{tabular}{|c|c|c|c|c|c|c|c|}
\hline CD1 & 365 & 0.40 & - & - & - & - & - \\
\hline CD2 & 357 & 2.28 & 421 & 448 & 27 & 71 & ND \\
\hline CD4 & 359 & 1.80 & 439 & 474 & 35 & 87 & 40.0 \\
\hline CD5 & 360 & 2.23 & 441 & 472 & 31 & 87 & 36.6 \\
\hline CD6 & 349 & 5.04 & 449 & 480 & 31 & 98 & 12.1 \\
\hline CD9 & 356 & 2.01 & 437 & 468 & 31 & 90 & 22.4 \\
\hline CD10 & 356 & 2.44 & 438 & 468 & 30 & 91 & 24.7 \\
\hline CD11 & 356 & 2.21 & 429 & 466 & 37 & 88 & 53.1 \\
\hline
\end{tabular}


The PL properties of CDs strongly depend on the precursors and preparative conditions, and reveal a complex internal and surface structure of these nanoparticles. Though there is still significant debate on the origin of the PL of CDs, several models have emerged that reflect the commonly observed features. ${ }^{50}$ One of the first approaches envisioned energy transfer between distinct states associated with the core, incorporating PAH domains, and the surface of the $\mathrm{CD}$ as the likely source of the observed optical properties. Another model was proposed that involves the formation of molecular fluorophores during the synthesis of the CDs (such as citrazinic acid and 2-pyridonebased molecules ${ }^{50-52}$ ) that significantly contribute to their emission. It thus appears that PL may result from combined contributions of PAH domains and molecular fluorophores, depending on the preparation procedure. For instance, at low synthesis temperature molecular fluorophores dominate the PL, while at high temperature they are more extensively carbonized and PL comes under the control of aromatic domains. ${ }^{52,53}$ All the herein described CDs displayed intrinsic fluorescence properties, except CD1 and CD7 that were produced at $100^{\circ} \mathrm{C}$. It is assumed that at such low temperature, neither PAH domains nor molecular fluorophores could form in significant amount. The UV absorption of the other CDs at $250 \mathrm{~nm}$ did not typically generate fluorescence as can be observed on the PL excitation spectrum for each nanoparticle (Fig. S3 $\dagger$ ). However in the lower-energy region, the spectra show a broad peak centered at $c a .440 \mathrm{~nm}$ that aggregates two more or less visible peaks centered at 430 and $450 \mathrm{~nm}$, except for CD2. In this case, the excitation peak was sharper and centered at $421 \mathrm{~nm}$, with no significant contribution around $450 \mathrm{~nm}$. This is an indication that the excitation peak at $c a .450 \mathrm{~nm}$ only appears when applying harsher reaction conditions during the CD synthesis (i.e., either $T>180^{\circ} \mathrm{C}$, or solvent-free reaction, or extended reaction time). Two secondary features may be observed at 370 and $305 \mathrm{~nm}$ that were attenuated for CDs processed at high temperature for an extended period of time (CD6). This is consistent with the model of molecular fluorophores that may be extensively carbonized at high temperature. Once again CD2 came out with no excitation band at $370 \mathrm{~nm}$, which suggests that synthesis of molecular fluorophores did not extensively proceed at $180{ }^{\circ} \mathrm{C}$ under solvothermal conditions. At $370 \mathrm{~nm}$, due to significant overlap of the excitation features, both the fluorophore molecules and the $\mathrm{CD}$ core and edge states are excited, though resulting in low intensity emission. At $430-450 \mathrm{~nm}$, molecular fluorophore absorption and emission are very low, while surface-state transitions of CDs dominate which are responsible for the brightest emission in CDs. The main features of the PL properties of the CDs described herein are summarized in Table 2. PL emission follows the Stokes type emission; namely, the PL emission wavelength is longer than the excitation wavelength. The Stokes shift for CDs fluorescence ranged between 23 and $37 \mathrm{~nm}$, which is beneficial for the distinction of the target from the background signal when used in fluorescent bio-imaging. With respect to the full width half maximum (FWHM) of the emission peak, values lie between 87 and $91 \mathrm{~nm}$ for all the CDs, except for CD2 (71 nm) and CD6 (98 nm). The narrowing of FWHM in CD2 is consistent with more unified chromophores produced under the milder reaction conditions. ${ }^{54}$ At the opposite, pyrolysis of the organic matter at higher temperature for an extended period of time is expected to produce a wider variety of chromophores, resulting in a broadening of the emission peak as was observed for CD6. The CDs show efficient but variable PL emission and can reach a highest quantum yield (QY) of $c a$. 63\%. Such high QYs are generally ascribed to the high nitrogen content of the CDs, as N-containing groups are excellent auxochromic moieties. $^{43,55}$ However, no correlation could be established herein with elemental composition (Table S1 $\dagger$ ), most likely revealing that not only the amount but also the nature of N-containing groups (pyridinic, pyrrolic, graphitic, amide, nitro...) in the CD structure have variable impact on the PL QY. ${ }^{56,57}$ Additionally, the as-produced CDs exhibit excitation-dependent emission, as shown in Fig. $\mathrm{S} 3, \dagger$ which is an intrinsic property of CDs that was attributed to the existence of different sizes or different light emitting sites on the surface of the nanoparticles..$^{13,47,58}$

\subsection{In vitro gene delivery and cytotoxicity}

The CDs were evaluated for their ability to promote gene delivery to mammalian cells in vitro. Experiments were performed on the A549 human lung carcinoma alveolar epithelial cell line, using a plasmid DNA encoding the luciferase protein from Gaussia princeps as reporter gene. Gaussia luciferase catalyzes a reaction with its substrate, coelenterazine, to produce light. Gene transfer efficiency thus can be conveniently determined by bioluminescence measurements. The level of luciferase expression was determined for each CD sample, varying the $\mathrm{CD} / \mathrm{pDNA}$ weight to weight (w/w) ratio (Fig. 4). For comparison, bPEI25k and bPEI600 were evaluated in parallel. As expected, bPEI25k mediated high gene transfer, and the optimal $\mathrm{w} / \mathrm{w}$ ratio was determined to be around 4 . At the opposite, no transfection signal was obtained with bPEI600. This was ascribed to the poor stability of the complexes the low
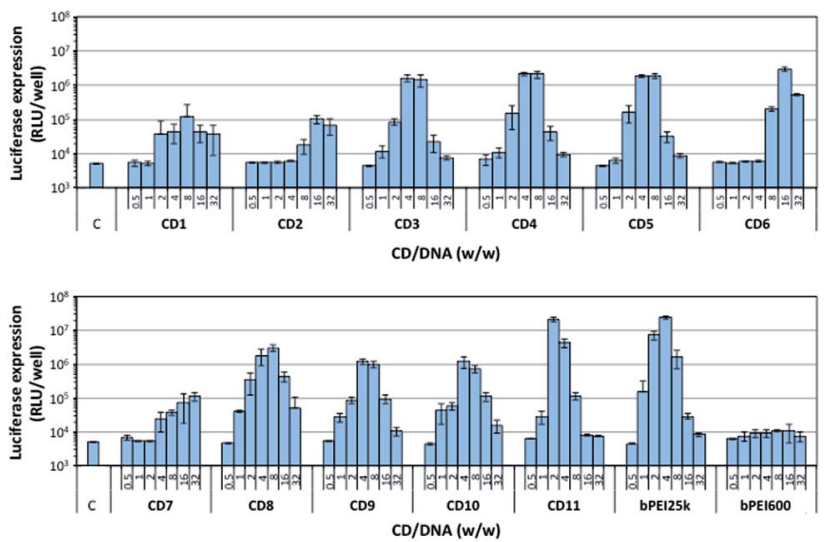

Fig. 4 Transfection efficiency of the various CDs described in this work as a function of $\mathrm{CD} / \mathrm{pDNA} \mathrm{w} / \mathrm{w}$ ratio (from 0.5 to 32 ). bPEl25k and bPEI600 were introduced for comparison. A549 cells were treated as described in the Experimental section and transfection rate was determined after $24 \mathrm{~h}$ incubation. $\mathrm{C}$ stands for control untreated cells. Data shown are representative of triplicate determinations (mean \pm $\mathrm{SD})$. 
molecular weight polyamine may form with DNA. Indeed, nucleic acid is thus imperfectly condensed and does not form discrete particles as required for cell internalization by endocytosis routes. Furthermore, residual negative charges at the surface of the complexes may prevent their interaction with the negatively charged plasma membrane, which is another prerequisite for efficient endocytosis. CDs prepared from citric acid and bPEI600 under solvothermal conditions and conventional heating (CD1 and CD2) failed as well to mediate significant transfection, luciferase bioluminescence signal being hardly an order of magnitude higher than that of untreated cells (background noise level). CDs produced by pyrolysis of the materials under atmospheric pressure (CD3-CD5) displayed a very similar transfection profile with optimum transfection efficiency at a w/w ratio of $c a$. 4 to 8 . Their transfection signal however was an order of magnitude lower than the one elicited by bPEI25k. Overheating under these conditions produced CDs (CD6) with degraded transfection efficiency. High transfection rate however could be obtained by increasing the amount of CDs in the complexes to a CD/pDNA w/w ratio of 16 . Microwave heating of citric acid and bPEI600 for 3 min under solvent-free conditions produced efficient gene transfer reagents provided the temperature was brought to $180{ }^{\circ} \mathrm{C}$ (CD8). Increasing the temperature did not show beneficial for transfection efficiency that was reduced by a $c a$. four-fold factor for CDs prepared at $210{ }^{\circ} \mathrm{C}$ (CD9) and $225^{\circ} \mathrm{C}$ (CD10). The higher transfection rate however was attained with reduced $\mathrm{CD} / \mathrm{pDNA} \mathrm{w} / \mathrm{w}$ ratio in these cases. Microwave heating at low temperature and for an extended period of time of the starting materials in solution $\left(100{ }^{\circ} \mathrm{C}, 30 \mathrm{~min}, \mathrm{CD} 7\right)$ failed to produce CDs with high transfection efficiency, even at the higher $\mathrm{CD} / \mathrm{pDNA}$ w/w ratio investigated. Finally, pyrolysis under microwave heating at normal pressure produced CD11 that allowed a transfection rate similar to that obtained with the gold standard gene delivery reagent bPEI25k. Furthermore, the optimal CD/pDNA w/w ratio shifted down to 2 while it was $c a$. 4 with bPEI25k. This is especially valuable considering the intrinsic toxicity of bPEI and related compounds.

In order to address the toxicity issue, we determined the viability of A549 cells exposed to the CD/pDNA complexes (Fig. 5) as well as to selected "bare" CDs (Fig. S4†). This was realized using a standard MTT assay based on mitochondrial activity measurements. The results revealed that CDs that were only poorly active as transfection reagents did not show any toxicity, even at the higher concentration tested (CD1, CD2, and CD7). Similarly, cells fully retained their mitochondrial activity when exposed to bPEI600/pDNA complexes. For CDs that were able to elicit a significant transfection response, associated cytotoxicity was invariably found. In most of the cases however, more than $77 \%$ mitochondrial activity still could be measured at the $\mathrm{CD}$ concentration that revealed optimal for gene delivery (CD3: 88\%; CD4: 86\%; CD5: 95\%; CD6: 77\%; CD9: 85\%; CD10: 79\%; CD11: 80\%). Noteworthy, all the CDs evaluated herein revealed significantly less harmful to cells than bPEI25k. Especially, cells treated with CD11/pDNA complexes retained up to $80 \%$ of their mitochondrial activity with maximum
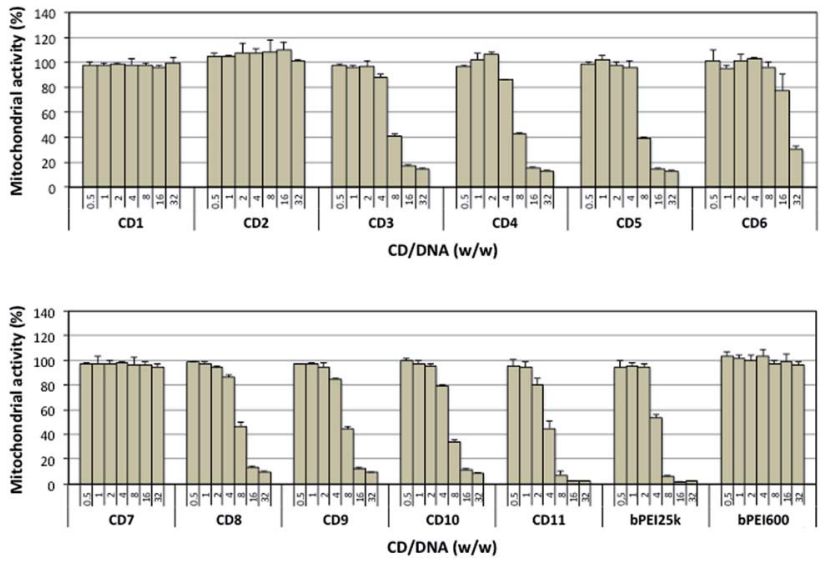

Fig. 5 Cytotoxicity of the various CD/pDNA complexes after incubation of A549 cell for $24 \mathrm{~h}$ as measured by the MTT assay. Results are reported as \% mitochondrial activity based on the control untreated cells normalized to $100 \%$. Data shown are representative of triplicate determinations (mean $\pm \mathrm{SD}$ ).

expression of the transgene while cells treated with bPEI25k/ pDNA only preserved $53 \%$ integrity at a similar transfection rate.

\section{Conclusions}

The influence of the carbonization procedure used to synthesize cationic CDs from a 1/4 (w/w) citric acid-bPEI600 mixture on the properties of the resulting nanoparticles has been investigated into details. Depending on the reaction conditions (temperature, solvent) and activation mode (conventional heating, microwave activation), CDs were produced with yields varying from 3 to $66 \%$. The size of the CDs was homogeneously found between 10 and $20 \mathrm{~nm}$ and their charge ranged from +22.5 to $+40.7 \mathrm{mV}$. No correlation could be established between the $\zeta$ potential value and the nitrogen content of the nanoparticles revealing variations of nitrogen distribution at the surface and within the core of the particles. With regard to their PL properties, the CDs displayed maximum excitation at 420$440 \mathrm{~nm}$ with emission around 450-480 $\mathrm{nm}$ and quantum yield up to $63 \%$. Despite their similar physical properties, the CDs exhibited marked differences in their gene delivery efficiency, as evaluated in the airway epithelial cells A549 using a pDNA encoding the Gaussia luciferase. CDs produced under microwave irradiation in a domestic oven thus revealed superior to others and compared to the gold standard transfection reagent bPEI25k, with an optimal CD/pDNA w/w ratio that was significantly down shifted, as was the associated cytotoxicity.

\section{Conflicts of interest}

The authors declare that they have no competing interests.

\section{Acknowledgements}

The authors are grateful for financial support from Agence nationale de sécurité sanitaire de l'alimentation, de 
l'environnement et du travail-ANSES (no. 2015/1/005), Canceropôle Grand Est (no. 16/416/S40), and Fondation ARC pour la recherche sur le cancer (no. PJA201512003488).

\section{Notes and references}

1 X. Y. Xu, R. Ray, Y. L. Gu, H. J. Ploehn, L. Gearheart, K. Raker and W. A. Scrivens, J. Am. Chem. Soc., 2004, 126, 1273612737.

2 Thomson Reuters, ISI Web of Knowledge, June 2018.

3 A. L. Himaja, P. S. Karthik and S. P. Singh, Chem. Rec., 2015, 15, 595-615.

4 S. Y. Lim, W. Shen and Z. Q. Gao, Chem. Soc. Rev., 2015, 44, 362-381.

5 P. L. Zuo, X. H. Lu, Z. G. Sun, Y. H. Guo and H. He, Microchim. Acta, 2016, 183, 519-542.

6 R. Wang, K. Q. Lu, Z. R. Tang and Y. J. Xu, J. Mater. Chem. A, 2017, 5, 3717-3734.

7 K. Hola, Y. Zhang, Y. Wang, E. P. Giannelis, R. Zboril and A. L. Rogach, Nano Today, 2014, 9, 590-603.

8 A. D. Zhao, Z. W. Chen, C. Q. Zhao, N. Gao, J. S. Ren and X. G. Qu, Carbon, 2015, 85, 309-327.

9 N. Duran, M. B. Simoes, A. C. M. de Moraes, W. J. Favaro and A. B. Seabra, J. Biomed. Nanotechnol., 2016, 12, 1323-1347.

10 F. L. Yuan, S. H. Li, Z. T. Fan, X. Y. Meng, L. Z. Fan and S. H. Yang, Nano Today, 2016, 11, 565-586.

11 Y. Du and S. J. Guo, Nanoscale, 2016, 8, 2532-2543.

12 K. Dimos, Curr. Org. Chem., 2016, 20, 682-695.

13 S. N. Baker and G. A. Baker, Angew. Chem., Int. Ed., 2010, 49, 6726-6744.

14 H. Li, Z. Kang, Y. Liu and S.-T. Lee, J. Mater. Chem., 2012, 22, 24230-24253.

15 B. F. Han, W. X. Wang, H. Y. Wu, F. Fang, N. Z. Wang, X. J. Zhang and S. K. Xu, Colloids Surf., B, 2012, 100, 209-214.

16 M. Hassan, V. G. Gomes, A. Dehghani and S. M. Ardekani, Nano Res., 2018, 11, 1-41.

17 H. Kim, S. Beack, S. Han, M. Shin, T. Lee, Y. Park, K. S. Kim, A. K. Yetisen, S. H. Yun, W. Kwon and S. K. Hahn, Adv. Mater., 2018, 30, 33.

18 Y. Y. Zhang, M. H. Wu, M. L. Wu, J. Y. Zhu and X. Zhang, ACS Omega, 2018, 3, 9126-9145.

19 C. Liu, P. Zhang, X. Zhai, F. Tian, W. Li, J. Yang, Y. Liu, H. Wang, W. Wang and W. Liu, Biomaterials, 2012, 33, 3604-3613.

20 P. Pierrat, R. R. Wang, D. Kereselidze, M. Lux, P. Didier, A. Kichler, F. Pons and L. Lebeau, Biomaterials, 2015, 51, 290-302.

21 S. Kim, Y. Choi, G. Park, C. Won, Y. J. Park, Y. Lee, B. S. Kim and D. H. Min, Nano Res., 2017, 10, 503-519.

22 J. Kim, J. Park, H. Kim, K. Singha and W. J. Kim, Biomaterials, 2013, 34, 7168-7180.

23 Y. F. Wu, H. C. Wu, C. H. Kuan, C. J. Lin, L. W. Wang, C. W. Chang and T. W. Wang, Sci. Rep., 2016, 6, 12.

24 S. Das, N. Debnath, Y. J. Cui, J. Unrine and S. R. Palli, ACS Appl. Mater. Interfaces, 2015, 7, 19530-19535.

25 H. J. Wang, J. Zhang, Y. H. Liu, T. Y. Luo, X. He and X. Q. Yu, RSC Adv., 2017, 7, 15613-15624.
26 X. Cao, J. P. Wang, W. W. Deng, J. J. Chen, Y. Wang, J. Zhou, P. Du, W. Q. Xu, Q. Wang, Q. L. Wang, Q. T. Yu, M. Spector, J. N. Yu and X. M. Xu, Sci. Rep., 2018, 8, e7057.

27 L. M. Hu, Y. Sun, S. L. Li, X. L. Wang, K. L. Hu, L. R. Wang, X. J. Liang and Y. Wu, Carbon, 2014, 67, 508-513.

28 Q. Dou, X. Fang, S. Jiang, P. L. Chee, T.-C. Lee and X. J. Loh, RSC Adv., 2015, 5, 46817-46822.

29 J. Zhou, W. W. Deng, Y. Wang, X. Cao, J. J. Chen, Q. Wang, W. Q. Xu, P. Du, Q. T. Yu, J. X. Chen, M. Spector, J. N. Yu and X. M. Xu, Acta Biomater., 2016, 42, 209-219.

30 X. D. Yang, Y. Wang, X. R. Shen, C. Y. Su, J. H. Yang, M. J. Piao, F. Jia, G. H. Gao, L. Zhang and Q. Lin, J. Colloid Interface Sci., 2017, 492, 1-7.

31 M. Zhang, X. Zhao, Z. Fang, Y. Niu, J. Lou, Y. Wu, S. Zou, S. Xia, M. Sun and F. Du, RSC Adv., 2017, 7, 3369-3375.

32 H. J. Wang, X. He, T. Y. Luo, J. Zhang, Y. H. Liu and X. Q. Yu, Nanoscale, 2017, 9, 5935-5947.

33 L. Wang, X. Wang, A. Bhirde, J. Cao, Y. Zeng, X. Huang, Y. Sun, G. Liu and X. Chen, Adv. Healthcare Mater., 2014, 3, 1203-1209.

34 S. Patnaik and K. C. Gupta, Expert Opin. Drug Delivery, 2013, 10, 215-228.

35 B. A. Tannous, Nat. Protoc., 2009, 4, 582-591.

36 G. A. Reynolds and K. H. Drexhage, Opt. Commun., 1975, 13, 222-225.

37 Y. Q. Dong, R. X. Wang, H. Li, J. W. Shao, Y. W. Chi, X. M. Lin and G. N. Chen, Carbon, 2012, 50, 2810-2815.

38 Y. Dong, R. Wang, G. Li, C. Chen, Y. Chi and G. Chen, Anal. Chem., 2012, 84, 6220-6224.

39 R. Wang, G. Li, Y. Dong, Y. Chi and G. Chen, Anal. Chem., 2013, 85, 8065-8069.

40 Y. Dong, R. Wang, W. Tian, Y. Chi and G. Chen, RSC Adv., 2014, 4, 3701-3705.

41 J. Liu, X. Liu, H. Luo and Y. Gao, RSC Adv., 2014, 4, 76487654.

42 C. Wang, Z. Xu and C. Zhang, ChemNanoMat, 2015, 1, 122127.

43 S. J. Zhu, Q. N. Meng, L. Wang, J. H. Zhang, Y. B. Song, H. Jin, K. Zhang, H. C. Sun, H. Y. Wang and B. Yang, Angew. Chem., Int. Ed., 2013, 52, 3953-3957.

44 F. M. Ye, C. F. Wu, Y. H. Jin, M. Wang, Y. H. Chan, J. B. Yu, W. Sun, S. Hayden and D. T. Chiu, Chem. Commun., 2012, 48, 1778-1780.

45 R. H. Terrill, T. A. Postlethwaite, C. H. Chen, C. D. Poon, A. Terzis, A. D. Chen, J. E. Hutchison, M. R. Clark, G. Wignall, J. D. Londono, R. Superfine, M. Falvo, C. S. Johnson, E. T. Samulski and R. W. Murray, J. Am. Chem. Soc., 1995, 117, 12537-12548.

46 R. Marega, V. Aroulmoji, F. Dinon, L. Vaccari, S. Giordani, A. Bianco, E. Murano and M. Prato, J. Am. Chem. Soc., 2009, 131, 9086-9093.

47 A. Kundu, J. Lee, B. Park, C. Ray, K. V. Sankar, W. S. Kim, S. H. Lee, I. J. Cho and S. C. Jun, J. Colloid Interface Sci., 2018, 513, 505-514.

48 E. Pretsch, P. Bühlmann and C. Affolter, Structure determination of organic compounds, Springer-Verlag, Berlin, 3rd edn, 2000. 
49 M. Sudolska, M. Dubecky, S. Sarkar, C. J. Reckmeier, R. Zboril, A. L. Rogach and M. Otyepka, J. Phys. Chem. C, 2015, 119, 13369-13373.

50 F. Ehrat, S. Bhattacharyya, J. Schneider, A. Lof, R. Wyrwich, A. L. Rogach, J. K. Stolarczyk, A. S. Urban and J. Feldmann, Nano Lett., 2017, 17, 7710-7716.

51 J. Schneider, C. J. Reckmeier, Y. Xiong, M. von Seckendorff, A. S. Susha, P. Kasak and A. L. Rogach, J. Phys. Chem. C, 2017, 121, 2014-2022.

52 W. Wang, B. Wang, H. Embrechts, C. Damm, A. Cadranel, V. Strauss, M. Distaso, V. Hinterberger, D. M. Guldi and W. Peukert, RSC Adv., 2017, 7, 24771-24780.

53 Y. Xiong, J. Schneider, C. J. Reckmeier, H. Huang, P. Kasák and A. L. Rogach, Nanoscale, 2017, 9, 11730-11738.
54 Y. P. Sun, B. Zhou, Y. Lin, W. Wang, K. A. S. Fernando, P. Pathak, M. J. Meziani, B. A. Harruff, X. Wang, H. F. Wang, P. J. G. Luo, H. Yang, M. E. Kose, B. L. Chen, L. M. Veca and S. Y. Xie, J. Am. Chem. Soc., 2006, 128, 7756-7757.

55 H. Tetsuka, R. Asahi, A. Nagoya, K. Okamoto, I. Tajima, R. Ohta and A. Okamoto, Adv. Mater., 2012, 24, 5333-5338.

56 B. C. M. Martindale, G. A. M. Hutton, C. A. Caputo, S. Prantl, R. Godin, J. R. Durrant and E. Reisner, Angew. Chem., Int. Ed., 2017, 56, 6459-6463.

57 S. Sarkar, M. Sudolska, M. Dubecky, C. J. Reckmeier, A. L. Rogach, R. Zboril and M. Otyepka, J. Phys. Chem. C, 2016, 120, 1303-1308.

58 Y. Q. Pang, H. Gao, L. H. Lai and X. L. Li, Mater. Res. Bull., 2018, 104, 83-86. 